\title{
The Computational Model of Firing Safety Limit for Naval Gun to Use Trajectory Correction Projectile Jiang $\mathrm{Hu}^{1, \mathrm{a}}$ and Qiang Gai ${ }^{1, \mathrm{~b}}$ \\ ${ }^{1}$ Department of equipment system, Dalian Naval Academy, Dalian, China \\ axiangjiangzhishui@163.com, biantingxueyuan@163.com
}

Keywords: Firing safety limit; Trajectory correction projectile; Dispersion errors; Firing support.

\begin{abstract}
During naval gun using trajectory correction projectile to fire on shore as oppressive weapon, it is necessary for attacking force to apart from the shore target and keep safety. According to characteristic of trajectory correction projectile, this paper discussed the influence factor of firing safety limit, and put forward the computational expressions of firing safety limit based on analytical method and simplified method respectively. This will be useful for the trajectory correction projectile to fire on shore.
\end{abstract}

\section{Introduction}

Trajectory correction projectile is a new type information ammunition which using trajectory correction fuze to correct trajectory deflection. It holds advantages of extended firing range, high hit target precision and high density of fire. In landing operation, trajectory correction projectile not only can strike at target directly, but also can offer firing cover and eliminate landing obstacle according to requirements of attacking force. During naval gun using trajectory correction projectile to fire on shore as oppressive weapon, it is necessary for attacking force to keep certain distance from firing target and ensure safety [1]. The above-mentioned distance between attacking force and target is firing safety limit.

The value of firing safety limit shouldn't be too small, otherwise it will endanger safety of attacking force. However, to enlarge the value of firing safety limit casually will bring difficulties for attacking force to strike at target. Therefore, the research on firing safety limit of trajectory correction projectile have significant realistic meaning.

\section{Influencing Factors}

During naval gun using trajectory correction projectile to fire on shore as oppressive weapon, main influencing factors of firing safety limit are firing data errors, dispersion errors, effective killing range of projectile and tilting terrain and so on.

Firing Data Errors. During trajectory correction projectile calculating shooting data, firing data errors are generated, mainly including target location error, ballistic and meteorological error, and working errors of correction mechanism and so on[2].

Trajectory correction projectile's firing data errors in distance and direction $\left(x_{n}, z_{n}\right)$ obeys two-dimensional normal distribution, whose distribution density function are as follows:

$$
\varphi\left(x_{n}, z_{n}\right)=\frac{\rho^{2}}{\pi E_{d} E_{f}} \exp \left\{-\rho^{2}\left[\frac{x_{n}^{2}}{E_{d}^{2}}+\frac{z_{n}^{2}}{E_{f}^{2}}\right]\right\}
$$

In the formula, $E_{d}$ and $E_{f}$ are probability errors in distance and direction of firing data errors, $\rho=0.4769362762$.

Dispersion Errors. Dispersion errors are mainly including servo system's errors of naval gun, initial disturbance errors, thrusting misalignment errors and ballistic coefficient errors and so on[3]. 
For dispersion errors in distance and direction of trajectory correction projectile $\left(x_{p}, z_{p}\right)$, its distribution density function is as follows:

$$
\varphi\left(x_{p}, z_{p}\right)=\frac{\rho^{2}}{\pi B_{d} B_{f}} \exp \left\{-\rho^{2}\left[\frac{x_{p}^{2}}{B_{d}^{2}}+\frac{z_{p}^{2}}{B_{f}^{2}}\right]\right\}
$$

In the formula, $B_{d}$ and $B_{f}$ are probability errors in distance and direction of dispersion errors.

Effective Killing Range of Projectile. Trajectory correction projectile damage targets mainly by projectile's explosive fragments, its effective killing range is related to target property, terrain environment, and fragment flying range and so on. Effective killing range of trajectory correction projectile in distance and direction are marked by $L_{x}$ and $L_{z}$.

Tilting Terrain. During firing on shore, because of tilting terrain, target may located on inclined plane toward shooting ship. We supposed ground tilting angle is $\alpha$, projectile drop angle is $\theta_{c}$, then on inclined plane, dispersion probability errors of trajectory correction projectile $B_{d}^{\prime}$ is as follows:

$$
B_{d}^{\prime}=\frac{B_{d} \cos \theta_{c}}{\cos \left(\theta_{c}+\alpha\right)}
$$

\section{Calculation Methods}

Analytical method and simplified method can be used to calculate firing safety limit of trajectory correction projectile. Analytical method is to confirm target damaging probability by numerical integration method, and take it as basis to calculate firing security limit.

Analytical Method. Supposed that the distance deviation between shooting-collimation position and attacking force was $a_{x}$, the direction deviation was $a_{z}$, and only the influence of dispersion errors was considered, the firing data errors were specific value $\left(x_{n}, z_{n}\right)$. The conditional probability for a round of trajectory correction projectile to hit attacking force is as follows:

$$
\begin{aligned}
& p\left(x_{n}, z_{n}\right)=\int_{a_{x}+x_{n}-L_{x}}^{a_{x}+x_{n}+L_{x}} \int_{a_{z}+z_{n}-L_{z}}^{a_{z}+z_{n}+L_{z}} \frac{\rho^{2}}{\pi B_{d}^{\prime} B_{f}^{\prime}} \exp \left\{-\rho^{2}\left[\frac{x^{2}}{B_{d}^{\prime 2}}+\frac{z^{2}}{B_{f}^{\prime 2}}\right]\right\} d x d z \\
& =\frac{1}{4}\left[\hat{\Phi}\left(\frac{a_{x}+x_{n}+L_{x}}{B_{d}^{\prime}}\right)-\hat{\Phi}\left(\frac{a_{x}+x_{n}-L_{x}}{B_{d}^{\prime}}\right)\right] \cdot\left[\hat{\Phi}\left(\frac{a_{z}+z_{n}+L_{z}}{B_{f}^{\prime}}\right)-\hat{\Phi}\left(\frac{a_{z}+z_{n}-L_{z}}{B_{f}^{\prime}}\right)\right]
\end{aligned}
$$

Assuming that average hit number which is needed to damage attacking force is $\omega$, then the conditional probability for a round of trajectory correction projectile to damage attacking force is as follows:

$$
R_{1}=\frac{P\left(x_{n}, z_{n}\right)}{\omega}
$$

The conditional probability that can't damage attacking force for several rounds of trajectory correction projectile is $\left[1-\frac{p\left(x_{n}, z_{n}\right)}{\omega}\right]^{N}$, then the conditional probability that can damage attacking force for several rounds of trajectory correction projectile is as below:

$$
R_{N}^{\prime}=1-\left[1-\frac{p\left(x_{n}, z_{n}\right)}{\omega}\right]^{N}
$$


If the firing data errors $\left(x_{n}, z_{n}\right)$ are giving all possible values, then the calculating formula of damage probability is as follows:

$$
R_{N}=\int_{-\infty}^{+\infty} \int_{-\infty}^{+\infty}\left\{1-\left[1-\frac{p\left(x_{n}, z_{n}\right)}{\omega}\right]^{N}\right\} \varphi_{n}\left(x_{n}, z_{n}\right) d x_{n} d z_{n}
$$

The Gauss-Hermite method can be used to calculate integration which is similar to formula (7). In the first place, the transformation is made as below:

$$
\left\{\begin{array}{l}
x_{1}=\rho \frac{x_{n}}{E_{d}} \\
x_{1}=\rho \frac{x_{n}}{E_{d}}
\end{array}\right.
$$

Then we may get the formula as follows:

$$
\begin{aligned}
& R_{N}=\int_{-\infty}^{+\infty} \int_{-\infty}^{+\infty}\left\{1-\left[1-\frac{p\left(x_{n}, z_{n}\right)}{\omega}\right]^{N}\right\} \varphi_{n}\left(x_{n}, z_{n}\right) d x_{n} d z_{n}=\frac{1}{\pi} \int_{-\infty}^{+\infty} \int_{-\infty}^{+\infty} e^{-\left(x_{1}^{2}+x_{2}^{2}\right)}\left\{1-\left[1-\frac{p\left(E_{d} x_{1} / \rho_{1}, E_{f} z_{1} / \rho_{1}\right)}{\omega}\right]^{N}\right\} d x_{1} d x_{2} \\
\approx & 1-\frac{1}{\pi} \sum_{i=1}^{n} A_{i} \sum_{j=1}^{n} A_{j}\left\{1-\left[1-\frac{p\left(E_{d} x_{1} / \rho, E_{f} z_{1} / \rho\right)}{\omega}\right]^{N}\right\}
\end{aligned}
$$

In the above formula, $n$ is the number of Gauss-Hermite nodes, $\xi_{i}, \quad \xi_{j}$ is the Gauss-Hermite quadrature nodes [4].

The safety probability $Q$, that means the attacking force can't be damaged, is used as quantitative indicator to determine firing safety limit. And the safety probability $Q=1-R_{N}$. Under the condition of direction deviation $a_{z}$ is certain value, so long as the value of $Q$ is given, then by constantly changing the value of $a_{x}$ and using numerical integration method, the value of distance safety limit can be obtained.

By use the same method, the value of direction safety limit $a_{z}$ can be worked out.

Simplified Method. When analytical method is used to calculate firing safety limit of trajectory correction projectile, though it has rather high accuracy, the calculation process is complex and it must with the help of computer programming. So the approximate formula is required to calculate firing safety limit. Not only the approximate formula can meet the accuracy requirement, but also it holds the merit of simple formula and short computation time.

Supposed that comprehensive probability error of distance and direction for trajectory correction projectile to fire on shore are $E_{x}$ and $E_{z}$, and the influence of tilting terrain, then the calculating formula can be get as follows:

$$
\left\{\begin{array}{l}
E_{x}=\sqrt{E_{d}^{2}+B_{d}^{\prime 2}} \\
E_{z}=\sqrt{E_{f}^{2}+B_{f}^{2}}
\end{array}\right.
$$

According to distribution law of shell's falling point, the approximate formula to calculate firing safety limit is as below:

$$
\left\{\begin{array}{l}
a_{x}=3 E_{x}+L_{x}-\frac{1}{2} L_{d} \\
a_{z}=3 E_{z}+L_{z}-\frac{1}{2} L_{f}
\end{array}\right.
$$

In the above formula, $L_{d}$ is the depth of target, $L_{f}$ is the width of target. 


\section{Application Analysis}

Supposed that trajectory correction projectile is used by naval gun during firing on shore, the depth of target $L_{d}$ and the width of target $L_{f}$ are both $40 \mathrm{~m}$, then the shooting distance is $d_{1}$ and $d_{2}$. Then we can get the change relationship between inclined plane's ground tilting angle $\alpha$ and distance safety limit $a_{x}$ as Fig. 1.

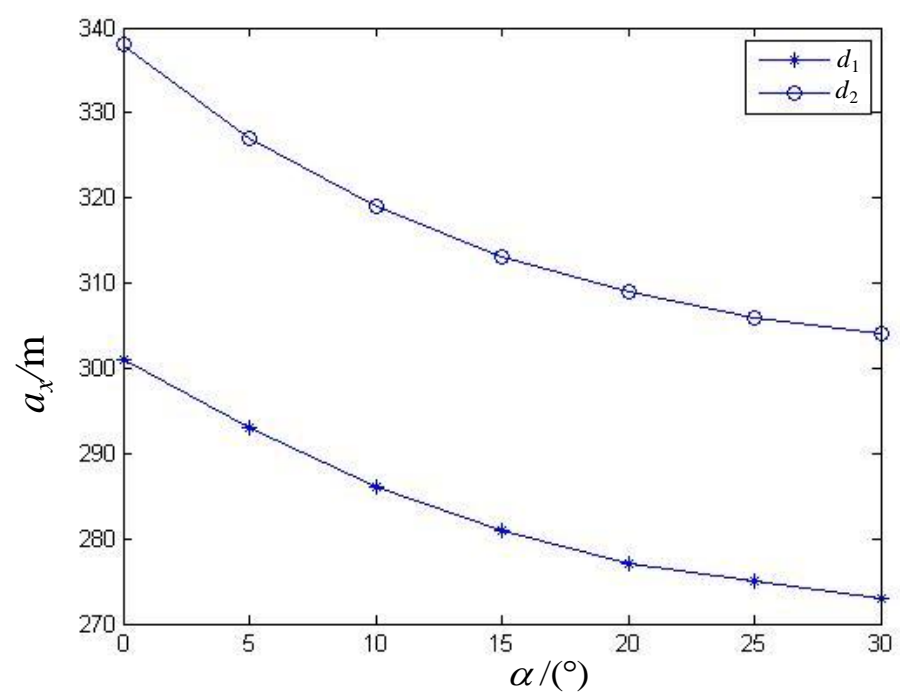

Figure 1. The change relationship between $a_{x}$ and $\alpha$

From the data of figure, we can see that, when the inclined plane's ground tilting angle $\alpha$ changes, distance safety limit $a_{x}$ changes obviously. Therefore, In the process of calculating firing safety limit, the influence of tilting terrain can't be ignored.

\section{Conclusion}

During naval gun using trajectory correction projectile to fire on shore as oppressive weapon, in order to guarantee safety of attacking force, it is necessary to use most accurate method to decide firing data [5]. Meanwhile, it is also necessary to consider firing errors, fragments' killing range and so on to confirm proper firing safety limit. Efforts to give same considerations on avoiding accidental injury of attacking force and completing attack task are preferred. This paper aimed at research on firing safety limit of trajectory correction projectile, and can give scientific basis for commander to make decision during firing on shore.

\section{References}

[1] Yuchen Zhang, Zhonghua Du. Calculation method of range correction for trajectory correction projectile. Computer and Simulation. Vol. 30 (2013) No. 6, p. 24-28.

[2] Yixin Liu, Firing efficiency of gun. Ordnance Industry, 2000, pp. 48-52.

[3] Yongqiang Ma. Launch process simulation of naval gun. Ship Electronic Engineering[J], Vol. 68 (2007) No.1, p. 58-65.

[4] Yongwei Zhang, Suochang Yang. Fire distribution plan of trajectory correction projectile. Command Control \& Simulation[J], Vol. 35 (2013) No.1, p. 65-70.

[5] Yanchun Shu. Method for correcting firing errors of trajectory correction projectile. Sichuan ordnance Journal[J], Vol. 36 (2015) No.2, p. 1-3. 